Revue internationale P.M.E.

Économie et gestion de la petite et moyenne entreprise

Revue

internationale

PME

\title{
GRH en PME
}

\section{Universalité et contingences : essai de théorisation}

\section{Henri Mahé De Boislandelle}

Volume 11, numéro 2-3, 1998

URI : https://id.erudit.org/iderudit/1009041ar

DOI : https://doi.org/10.7202/1009041ar

Aller au sommaire du numéro

\section{Éditeur(s)}

Presses de l’Université du Québec

ISSN

0776-5436 (imprimé)

1918-9699 (numérique)

Découvrir la revue

\section{Citer cet article}

Mahé De Boislandelle, H. (1998). GRH en PME : universalité et contingences : essai de théorisation. Revue internationale P.M.E., 11(2-3), 11-30.

https://doi.org/10.7202/1009041ar

\section{Résumé de l'article}

L'observation des pratiques de gestion des ressources humaines en PME conduit à constater une très grande diversité de situations concernant la taille, l'activité, les caractéristiques organisationnelles et la personnalité du dirigeant, mais aussi à relever des spécificités. Pour systématiser cet état, nous proposons un modèle contingentiel intégrant les principaux déterminants. Parmi ceux-ci, on note les variables contextuelles de l'organisation (caractéristiques structurelles, stratégiques et sociales), les variables environnementales (liées au contexte sociétal, technico- économique et à la conjoncture économique) ainsi que les caractéristiques personnelles du dirigeant. Chez ce dernier, on souligne un manque de référence à un modèle global de régulation intégrant explicitement la GRH, un comportement spécifique au travail ainsi qu'un certain nombre de biais cognitifs récurrents (effet de microcosme, effet de grossissement, etc.). Plus généralement, on observe une formalisation croissante avec la taille, une professionnalisation liée à l'importation de méthodes et l'émergence de procédures induites par les modes de coopération avec d'autres entreprises.
Ce document est protégé par la loi sur le droit d'auteur. L'utilisation des services d’Érudit (y compris la reproduction) est assujettie à sa politique d'utilisation que vous pouvez consulter en ligne.

https://apropos.erudit.org/fr/usagers/politique-dutilisation/ 


\title{
GRH en PME Universalité et contingences: essai de théorisation
}

\author{
Henri MAHÉ DE BOISLANDELLE \\ Université de Montpellier I
}

\section{MOTS CLÉS}

\section{GRH - PME - Contingence - Déterminant - Mix social Profil de dirigeant - Biais cognitif - Effet de grossissement Effet de microcosme - Théorisation}

\begin{abstract}
RÉSUMÉ
L'observation des pratiques de gestion des ressources humaines en PME conduit à constater une très grande diversité de situations concernant la taille, l'activité, les caractéristiques organisationnelles et la personnalité du dirigeant, mais aussi à relever des spécificités. Pour systématiser cet état, nous proposons un modèle contingentiel intégrant les principaux déterminants. Parmi ceux-ci, on note les variables contextuelles de l'organisation (caractéristiques structurelles, stratégiques et sociales), les variables environnementales (liées au contexte sociétal, technicoéconomique et à la conjoncture économique) ainsi que les caractéristiques personnelles du dirigeant. Chez ce dernier, on souligne un manque de référence à un modèle global de régulation intégrant explicitement la $G R H$, un comportement spécifique au travail ainsi qu'un certain nombre de biais cognitifs récurrents (effet de microcosme, effet de grossissement, etc.). Plus généralement, on observe une formalisation croissante avec la taille, une professionnalisation liée à l'importation de méthodes et l'émergence de procédures induites par les modes de coopération avec d'autres entreprises.
\end{abstract}

\section{L'AUTEUR}

Henri Mahé de Boislandelle est professeur en sciences de gestion à l'Université de Montpellier I (Faculté d'administration et gestion). Il est l'auteur de travaux et publications sur le management des PME et la gestion des ressources humaines. II dirige une équipe de recherche spécialisée dans les pratiques de GRH en PME. II est en contact avec différentes organisations dans le cadre d'activités d'études. Il a publié, en 1998, Dictionnaire de gestion: Concepts, Vocabulaires, Outils, chez Economica.

Adresse : Université de Montpellier I, Espace Richter, avenue de la Mer, BP 5640, 34054 Montpellier Cedex I, Télécopieur : 04-67-15-85-56 


\begin{abstract}
When considering the management of human resources in SMEs, we do not simply notice a large variety of situations due to size, sector of activity, organization and personality of the manager, but we also find specificities. In order to systematize such a situation, we propose a contingent model integrating the basic determinants. Among these, we can notice the variables linked to the organization itself (structural, strategic and social characteristics), those linked to the environment (society, technological and economic context, economic situation) and the main features of the manager's personality. As far as the latter is concerned, we can stress the nonreference to a global model of management clearly integrating HRM, a specific work behavior and a certain number of recurrent cognitive distortions (microcosm effect, expansion effect, etc.). More generally, we observe a formalization which increases with size, a professionalization due to imported methods and the emergence of processes deriving from the various ways of cooperating with other companies.
\end{abstract}

\title{
RESUMEN
}

La observación de las prácticas de gestión de recursos humanos en las PyMEs lleva a constatar una gran diversidad de situaciones en lo que respecta al tamaño, a la actividad, a las características de organización y a la personalidad del directivo. Esta diversidad de situaciones es fuente de especificidades. Para sistematizar tal estado de cosas proponemos un modelo contingencial que integra los principales determinantes. Entre ellos destacan las variables contextuales de la organización (características estructurales, estratégicas y sociales) y las variables ambientales (vinculadas al entorno societal o tecnicoeconómico y a la coyuntura económica), así como las características personales del directivo. En cuanto a este último se refiere, se recalca una falta de referencia a un modelo global de regulación que integra explícitamente la GRH y un comportamiento específico en el trabajo, así como ciertas "ópticas" recurrentes (efecto de microcosmos, efecto de ampliación, etc.). De manera más general, se observa una formalización que crece con el tamaño, una profesionalización vinculada a la importación de métodos y la emergencia de herramientas generadas por los modos de cooperación con otras empresas.

\section{Introduction}

La PME n'est pas une catégorie homogène, mais une appellation commode. Elle désigne de façon simplificatrice une réalité multiple, celle des entreprises ayant un effectif compris entre 10 et 500 salariés susceptibles de se différencier par la taille, par l'activité, par la forme de propriété et par les modes de gestion ${ }^{1} \ldots$

L'amélioration de l'efficacité de la GRH en PME est un enjeu d'importance, car les PME sont nombreuses, pourvoyeuses d'emplois et souvent reconnues

1. La définition retenue par la CGPME (France) précise de façon complémentaire d'autres aspects : «Les PME sont celles dans lesquelles les chefs d'entreprise assurent personnellement et directement des responsabilités financières, techniques, sociales et morales de l'entreprise, quelle que soit sa forme juridique .» 
comme mieux adaptées aux besoins de changement requis par les évolutions technologiques, économiques, politiques et sociales de notre fin de siècle et probablement du siècle suivant.

La démarche de théorisation est difficile et périlleuse, car il s'agit surtout de saisir la diversité et le contingent. Il ne peut donc être question de proposer un modèle théorique général, unique et universel, mais plutôt d'en proposer plusieurs ou mieux encore de suggérer un cadre général d'appréhension susceptible d'intégrer différents facteurs de contingence ou de détermination du système de GRH, aussi bien internes qu'externes à l'organisation. Au-delà de ce cadrage général des déterminants amonts du système de GRH, il est également souhaitable d'identifier les caractéristiques dominantes des pratiques de GRH offrant des modalités relativement invariantes. En d'autres termes, il faut essayer de préciser les paramètres distinctifs susceptibles d'aider à la théorisation ou modélisation du management des ressources humaines en PME. Autrement dit, admettre l'idiosyncrasie de chaque entreprise (ses spécificités) et dans le même temps la relativiser par rapport à des caractéristiques largement communes.

L'intérêt en est considérable puisque cela peut permettre, par le biais d'un détour théorique, d'améliorer les modes de gestion actuels et futurs, c'est-à-dire la production d'outils, la définition de procédures et l'adoption de certains comportements exigés par ces types de structures.

Pour en donner la mesure, nous proposons de brosser à grands traits les deux volets de cette démarche en présentant, d'une part, les déterminants de la GRH en PME ou les facteurs de contingence et, d'autre part, les caractéristiques dominantes des systèmes de GRH en PME ou paramètres distinctifs.

\section{Les déterminants de la GRH en PME ou facteurs de contingence}

Depuis $1988^{2}$, nous avons eu l'occasion de suggérer quelques modèles généraux de déterminants et observé des propositions d'autres auteurs cherchant à les enrichir ou allant dans le même sens.

La poursuite de la réflexion complétée par des observations de terrain nous conduit aujourd'hui à réutiliser le cadre général de cette modélisation en l'améliorant toutefois par l'insertion d'un plus grand nombre de données explicatives et surtout par le recours à des variables mieux ciblées.

2. Année de parution de la première édition de Gestion des ressources humaines dans les $P M E$, chez Economica. 
FIGURE 1

\section{Cadre contingentiel de la GRH en PME} (déterminants internes et externes)

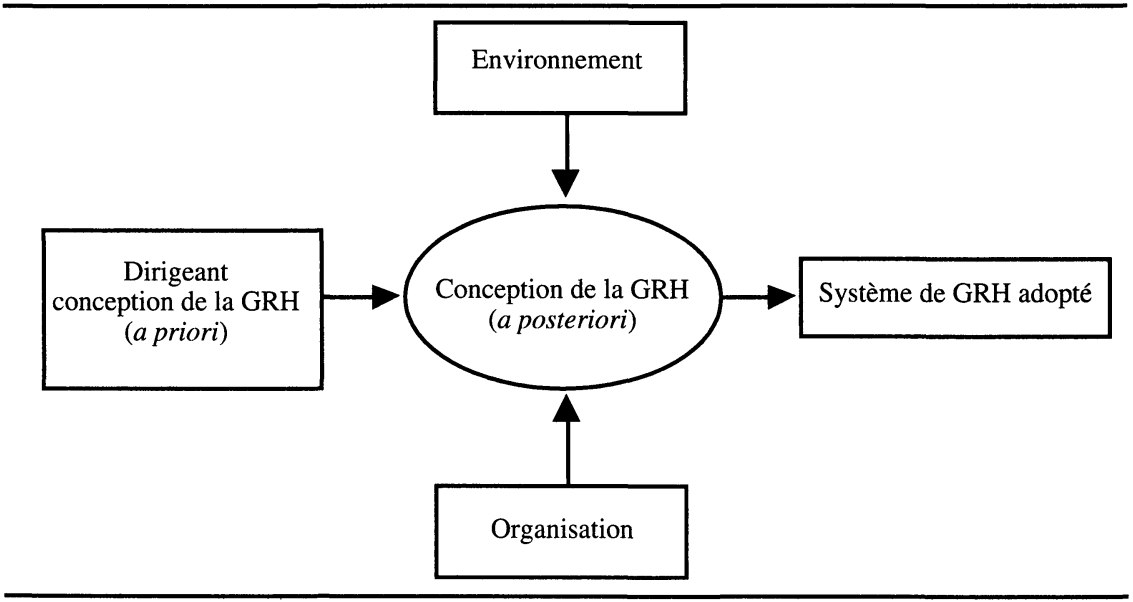

Partant de ce schéma à caractère général, lequel relève les influences principales, il importe d'effectuer quelques gros plans pour donner plus de précision aux contenus respectifs des trois déterminants, mais aussi au sens accordé à la conception a priori et à la conception a posteriori (après incidence des différents facteurs d'influence). C'est ce que nous allons voir en détaillant plus complètement les variables contextuelles de l'organisation, les variables environnementales et les caractéristiques personnelles du dirigeant.

\subsection{La conception a priori et la conception a posteriori de la GRH}

Dans le schéma 1 ci-dessus, nous avons distingué volontairement une conception a priori et une conception a posteriori pour souligner le fait qu'un système reste rarement imperméable aux sollicitations des ensembles qui l'entourent.

La conception a priori de la GRH et du management appartient en propre au dirigeant. Par cela, il faut entendre sa démarche hors contexte résultant de sa personnalité, de sa formation, de ses modèles de référence (explicites ou implicites), de ses antécédents et de son sens de la relation aux autres, etc. Nous prenons ici en considération la place qu'il accorde à la dynamique des hommes, à la connaissance des motivations et des capacités humaines dans une organisation et à la mobilisation des énergies. 
Cette conception a priori, quelle que soit sa force, ne peut rester étanche au contexte interne et externe. En effet, des interactions des deux grandes composantes structurantes que sont l'organisation et l'environnement émergera sans aucun doute une autre conception de la GRH ${ }^{3}$ que nous qualifions de «a posteriori » qui sera la résultante de rapports de force entre les parties concernées (salariés et dirigeants) et d'autres facteurs d'influence de natures diverses (juridiques, sociales, techniques, etc.).

Nous entendons ainsi par conception a posteriori que le dirigeant, même s'il est l'acteur principal de la politique de GRH en PME, ne pourra concevoir ni développer une politique de GRH abstraite de tout contexte interne et externe.

Les structures et les stratégies organisationnelles tout autant que les structures et les variables environnementales feront évoluer la conception de la GRH dans un sens plus ou moins favorable au personnel et à l'entreprise en termes de performances (sociales et économiques). Par exemple, en termes environnementaux, une nouvelle législation sociale plus favorable au salarié affectera le plus souvent les coûts. Inversement, un marché du travail marqué par le sous-emploi affectera la GRH dans un sens moins favorable au personnel.

Des sollicitations ou propositions de conseils en GRH et suggestions faites aux dirigeants par des pairs professionnels, dans le cadre de structures patronales de formation ou d'aide à la résolution de problèmes, influenceront les méthodes et les outils de la GRH.

Au plan organisationnel, il est facile d'imaginer que la structure et la dynamique du personnel en relation dialectique avec le dirigeant induiront des processus de compromis / négociation selon le mode de représentation du personnel et que l'état des rapports de force entre la direction et les salariés affectera les politiques de GRH, autrement dit, les choix.

FIGURE 2

Dialectique dirigeant/personnel (fondement de la GRH)

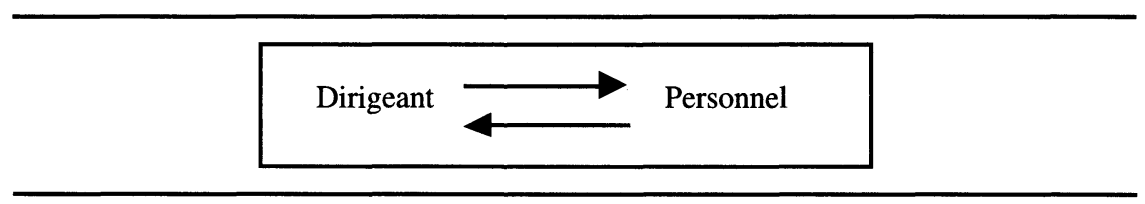

Il résultera de toutes ces incidences :

- une politique d'emploi plus ou moins précaire ;

3. Et du management qui sont étroitement liés. 
- une politique de rémunération audacieuse ou à la traîne ;

- une politique de valorisation systématique ou modeste;

- une politique de participation (communication et participation au processus décisionnel) qui sera le reflet d'un contexte conflictuel ou consensuel, des caractéristiques structurelles (métiers, types de production, niveaux hiérarchiques, etc.) tout autant que des choix stratégiques (diversification, recentrage, etc.).

Dans tous les cas, les modalités de mise en œuvre (procédures, délégations, etc.) seront révélatrices de confiance ou de défiance à l'égard du personnel. L'attitude de confiance ou de défiance adoptée par le dirigeant constituera le fondement du système de GRH. Il sera donc important de l'identifier. Le choix des outils de GRH et notamment des systèmes de contrôle du personnel seront d'excellents révélateurs de l'attitude.

Il ressort de ce qui précède que de nombreux facteurs d'influence tant internes qu'externes induiront des politiques de personnel extrêmement variées, autrement dit, des mix sociaux spécifiques à chaque organisation. Ces mix sociaux combineront quatre domaines ${ }^{4}$ et se situeront à trois niveaux ${ }^{5} \mathrm{~d}^{\prime}$ action de façon simultanée.

\subsection{Les déterminants de la conception a posteriori de la GRH}

Par-delà le cadre général fixé précédemment, il est facile d'imaginer une très grande diversité de conceptions a posteriori de la GRH. Celles-ci pourront de surcroît être affectées par les caractéristiques personnelles d'acteurs internes ayant reçu délégation en matière de GRH (décideurs désignés) :

- soit au titre d'acteur fonctionnel (cela peut être le cas d'un responsable des ressources humaines tel qu'un DRH à temps plein ou à temps partiel doté de pouvoirs d'initiative plus ou moins étendus ou d'un responsable de formation);

- soit au titre d'acteurs appartenant à la ligne hiérarchique, managers, ayant reçu délégation et exerçant un rôle dans l'administration du personnel ou dans la mise en œuvre de procédures relevant des politiques de personnel (cela peut être le cas des $\mathrm{DAF}^{6}$ en PME);

4. Il faut entendre par mix social une combinaison d'actions de gestion portant simultanément sur quatre variables d'action principales de la GRH qui sont l'emploi, la rémunération, la valorisation et la participation.

5. Il faut entendre les niveaux administratifs, politiques et stratégiques de la GRH.

6. DAF : directeur administratif et financier. 
- soit au titre de salariés représentants du personnel, ou simples salariés, ayant pris des initiatives à l'occasion de négociations, de manifestations revendicatives ou de consultations en rapport avec la direction.

FIGURE 3

\section{Déterminants contingentiels de la GRH en PME (Acteurs et facteurs internes)}

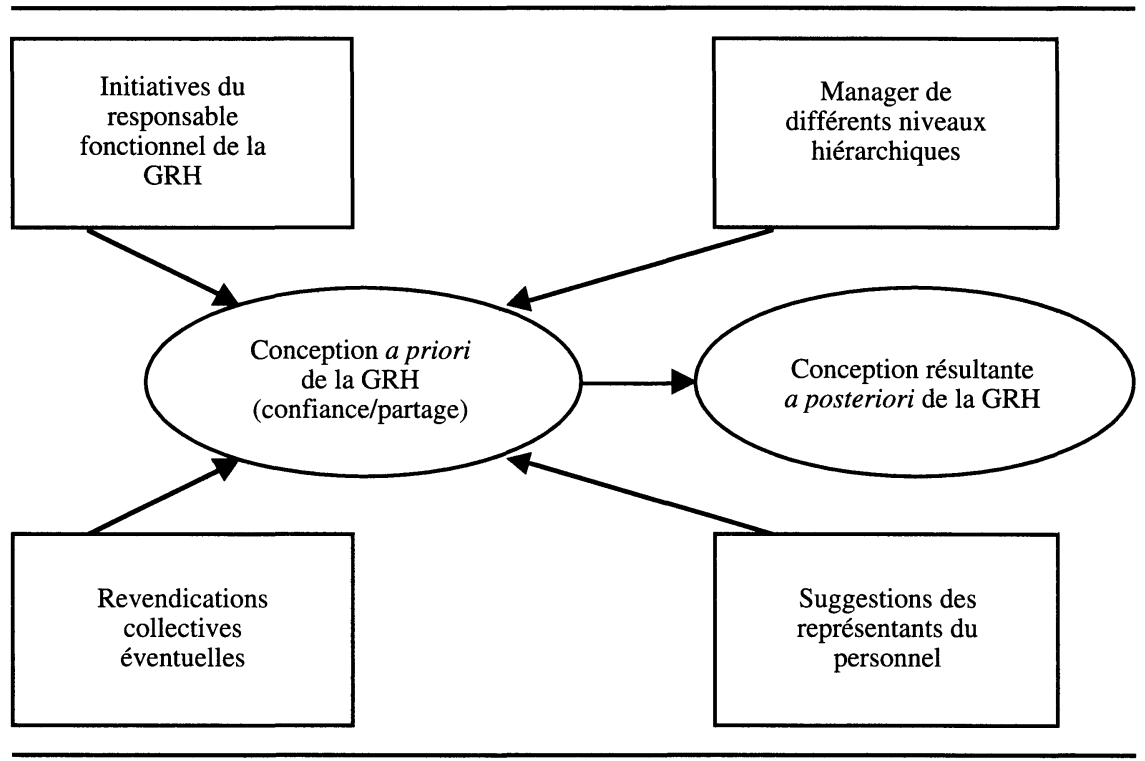

Après ces précisions sur les déterminants à dominante interne (acteurs et démarches) synthétisées dans le schéma 3 , explicitons plus complètement les autres types de déterminants de la GRH évoqués dans le schéma 1 en abordant successivement les variables contextuelles de l'organisation, les variables environnementales et les variables structurelles, attitudinales et comportementales relatives au dirigeant.

Parmi les variables contextuelles de l'organisation, on peut distinguer les caractéristiques structurelles, stratégiques et sociales. Le tableau 1 en résume l'essentiel.

Il apparaît, à l'évidence, que ces trois catégories de caractéristiques tenant aux aspects structurels, aux orientations stratégiques et au contexte social ne peuvent pas être sans incidence sur la politique de GRH tant en termes d'emploi que de rémunération, de valorisation et de participation.

Parmi les variables environnementales (extérieures au système entreprise), on peut distinguer les caractéristiques générales de la société au sein de laquelle opère l'entreprise, les données technologiques et économiques spécifiques à ses activités et, enfin, l'état du contexte concurrentiel et conjoncturel. 


\section{TABLEAU 1 \\ Variables contextuelles de l'organisation (caractéristiques structurelles, stratégiques et sociales)}

\begin{tabular}{|c|c|c|c|c|}
\hline \multicolumn{5}{|c|}{ Contexte organisationnel } \\
\hline & Caractéristiques structurelle & & Caractéristiques stratégiques & Caractéristiques sociales \\
\hline- & $\begin{array}{l}\text { Taille de l'organisation } \\
\text { (effectif, montant du capital, } \\
\text { volume d'affaires, etc.). } \\
\text { Localisation et étendue } \\
\text { géographique (siège social, } \\
\text { établissements, etc.). } \\
\text { Propriété du capital } \\
\text { (dirigeant propriétaire, } \\
\text { dirigeant salarié, filiale de } \\
\text { groupe, coopérative, etc.). } \\
\text { Âge de l'organisation } \\
\text { (ancienneté des activités, etc.). } \\
\text { Configuration organisa- } \\
\text { tionnelle (nombre de } \\
\text { niveaux hiérarchiques, } \\
\text { délégations, etc.). } \\
\text { Activités de l'entreprise } \\
\text { (production, distribution, } \\
\text { service, etc.). } \\
\text { Système de transforma- } \\
\text { tion de l'entreprise } \\
\text { (technologies et procédés } \\
\text { dominants). } \\
\text { Type de production (à la } \\
\text { commande, en série } \\
\text { standardisée, par lots, etc.). } \\
\text { Procédés et métiers. } \\
\text {.. }\end{array}$ & $\begin{array}{l}- \\
-\end{array}$ & $\begin{array}{l}\text { Orientations stratégiques } \\
\text { principales (diversification, } \\
\text { recentrage, croissance, } \\
\text { maintien, etc.). } \\
\text { Base de compétitivité } \\
\text { (service, coût, etc.). } \\
\text { Espace géographique de } \\
\text { référence (local, national, } \\
\text { global, etc.) visé. } \\
\text { Adaptation aux caractéris- } \\
\text { tiques de l'environnement } \\
\text { (attitude de veille } \\
\text { stratégique), vision de } \\
\text { l'organisation et de } \\
\text { l'environnement. } \\
\text { Projet(s) d'entreprise. } \\
\text { Domaines d'activité } \\
\text { stratégique (DAS). } \\
\text { Combinaison de cycle(s) } \\
\text { de développement parmi } \\
\text { plusieurs DAS. } \\
\text { Stratégies de dévelop- } \\
\text { pement de l'entreprise. } \\
\text { ‥ }\end{array}$ & $\begin{array}{ll}\text { - } & \begin{array}{l}\text { Structure des effectifs } \\
\text { (qualification, âge, }\end{array} \\
\text { ancienneté, etc.). } \\
\text { - } & \text { Représentation du } \\
\text { personnel (importance). } \\
\text { - } & \text { Militantisme (dynamisme, } \\
\text { passivité, etc.). } \\
\text { - } & \text { Histoire sociale de } \\
\text { l'organisation (dominantes } \\
\text { consensuelles ou conflic- } \\
\text { tuelles...). } \\
\text { - }\end{array}$ \\
\hline
\end{tabular}

\section{TABLEAU 2 \\ Variables environnementales (contexte sociétal, technico-économique et conjoncturel)}

\begin{tabular}{lll}
\hline Sociétal & \multicolumn{1}{c}{$\begin{array}{c}\text { Contexte environnemental } \\
\text { Technico-économique }\end{array}$} & Conjoncturel \\
\hline - Culture nationale, locale & - Technologies dominantes & - Dynamisme \\
- Droit, usages, coutumes & - Technologies spécifiques & - Déréglementation \\
- Institutions économiques & - Technologies spécifiques & - État de l'emploi \\
- Données macrosociales & - Taille(s) du (des) marché(s) & - Dynamisme concurrentiel \\
- Institutions politiques & - Structures économiques & - Ouverture des marchés \\
- Attentes, aspirations & - Conjoncture économique & - Stabilité /turbulence \\
collectives & $-\ldots$ & - Transparence /opacité \\
- ... & & - Complexité/simplicité \\
& & - Accessibilité /barrières \\
\hline
\end{tabular}


TABleau 3

Variables personnelles relatives au dirigeant (physiques, psychologiques, psychosociologiques et sociales)

\begin{tabular}{l} 
Caractéristiques structurelles, attitudinales et comportementales \\
\hline - Âge \\
- Santé du dirigeant \\
- Type de formation \\
- Niveau de formation \\
- Origine sociale \\
- Parcours professionnel \\
- Parcours personnel (situation familiale) \\
- Valeurs culturelles dominantes \\
- Système cognitif du dirigeant (intelligence, mémorisation, rapidité) \\
- Capacité d'analyse, de synthèse et de restitution \\
- Visions et intentions stratégiques \\
- Conceptions managériales et pratiques managériales \\
- Perception des niveaux de GRH (administratifs, politiques et stratégiques) \\
- Conception de la GRH a priori (modèles de GRH implicites ou explicites) \\
- Sensibilité aux influences internes et externes \\
- Style de direction (communication, délégation, centralisation, etc.) \\
- Forme(s) de leadership(s) mobilisé(s) \\
- Capacités d'adaptation \\
- Capacités d'anticipation \\
\hline
\end{tabular}

Parmi les variables personnelles du dirigeant ou de l'équipe dirigeante, on peut distinguer des caractéristiques physiques, psychologiques, psychosociologiques et sociales susceptibles d'avoir une incidence sur les pratiques ou sur les conceptions de la GRH ou du management des hommes.

Compte tenu du poids déterminant du dirigeant dans la PME, il convient de s'intéresser de façon plus approfondie à sa personnalité et à ses représentations mentales aussi bien dans leurs implications immédiates (outils et méthodes utilisés) que futures (visions à long terme, orientations stratégiques) ${ }^{7}$.

Précisons immédiatement, en raison de l'importance du propos, ce qu'il faut entendre par la construction subjective de sa propre réalité. Cette dernière devient ainsi la base référentielle du processus décisionnel et des orientations politiques qu'il adoptera.

De façon complémentaire et synthétique, l'identification du profil du dirigeant peut être effectuée par le recours à huit axes d'analyse particulièrement pertinents.

7. Se reporter, pour plus de précision, à notre article «L'effet de grossissement chez le dirigeant de PME » dans Gestion 2000, vol. 4, juillet-août 1996 ou encore à notre ouvrage Gestion des Ressources Humaines dans les PME, Economica, 1998, où nous consacrons un chapitre aux biais cognitifs et à leurs incidences sur le plan du management et de la GRH. 


\section{Axes de repérage du profil des dirigeants}

\begin{tabular}{|c|c|c|}
\hline Axe de l'action & (fonctionnement & développement) \\
\hline Axe de l'implication & (risque personnel faible & risque personnel fort) \\
\hline Axe de la méthodologie & (analyse logique & approche intuitive) \\
\hline Axe des représentations & (outils immédiats & visions dans la durée) \\
\hline Axe des valeurs & (idéologie collective & idéologie individuelle) \\
\hline Axe des relations & (confiance a priori & méfiance a priori) \\
\hline Axe de la propriété & (volonté de partage & refus de partage) \\
\hline Axe de la continuité & (profit à court terme & recherche de pérennité) \\
\hline
\end{tabular}

N.B. : Le fléchage $(\longleftrightarrow$ ) signifie que la position sur chaque axe de repérage se situe sur un continuum pouvant aller d'une position extrême à l'autre.

Il s'agit pour chacun d'un continuum marqué par des positions extrêmes. En pratique, il est facile de concevoir des échelles de gradation (de 4, 5, 6 ou 7 degrés) afin de différencier les états extrêmes et intermédiaires et d'opérationaliser le repérage.

Sur le premier axe (action), on pourra situer l'orientation vers la gestion au quotidien ou vers la volonté de transformation du système géré.

Sur le deuxième axe (implication), on repérera l'attitude par rapport à la prise de risque (prudence, audace).

Sur le troisième axe (méthodologie), on notera le système cognitif du dirigeant dominé par l'intuition ou le recours à des outils d'analyse (rationnels).

Sur le quatrième axe (représentations), on observera l'horizon de calcul qui sera privilégié (court, moyen, long terme).

Sur le cinquième axe (valeurs), on notera le caractère collectif ou individuel des objectifs poursuivis.

Sur le sixième axe (relations), on positionnera la hiérarchie des préférences sociales et relationnelles.

Sur le septième axe (propriété), on relèvera la volonté ou le refus de partage des gains monétaires et des pouvoirs résultant du « sentiment de propriété éprouvé ».

Sur le huitième axe (continuité), on distinguera la préférence pour le profit immédiat ou pour la pérennisation de l'organisation.

Par une combinaison graphique des positionnements sur les huit axes, avec l'aide d'échelles de Likert, il sera possible de faire émerger des profils types et éventuellement le profil singulier de chaque dirigeant. 
Il ressort ainsi de cette première partie que les déterminants du système de GRH, tant internes qu'externes, sont nombreux et complexes et qu'une place particulièrement importante doit être accordée au dirigeant. Examinons maintenant de façon plus approfondie les biais cognitifs affectant les diagnostics et les choix décisionnels du dirigeant ainsi que son comportement au travail. Des caractéristiques comportementales du dirigeant autant que de la taille de l'entreprise et des dépendances environnementales résulteront des modes de formalisation de la fonction ressources humaines en PME extrêmement variés.

\section{Les caractéristiques dominantes des systèmes de GRH en PME, ou paramètres distinctifs}

Nous basant sur différentes études empiriques portant sur les pratiques observées et notamment sur des études longitudinales (diachroniques ou historiques) approfondies de quelques entreprises, ainsi que sur des études synchroniques d'échantillons d'entreprises importants, nous en tirons ici quelques enseignements à caractère général.

Il s'agit de mettre en exergue les dominantes qui émergent le plus fréquemment en PME comparativement aux GE (grandes entreprises de plus de 500 salariés). Au-delà, il sera souhaitable de souligner certaines spécificités relatives aux PME. Parmi les plus notables, nous préciserons dans un premier temps le rôle fondamental du système cognitif du dirigeant, associé aux caractéristiques de son travail, et dans un second temps, nous examinerons l'émergence organisationnelle de la fonction en liaison, d'une part, avec la taille de l'organisation et, d'autre part, avec son réseau de dépendances amont ou aval.

\subsection{Référentiels de GRH, système cognitif et caractéristiques des activités du dirigeant}

La manière de penser autant que la nature et la diversité des activités que le dirigeant réalise ont une influence prépondérante sur sa manière d'appréhender la gestion des ressources humaines. Il importe donc de s'intéresser, d'une part, à ses principaux référentiels, à son système cognitif et biais de perception et, d'autre part, à la nature de ses activités.

\section{Référentiels de GRH chez le dirigeant}

Le dirigeant occupant une place de choix en PME, il est légitime de s'intéresser à ses représentations mentales en distinguant ses référentiels immédiats (outils, modèles, etc.) de ses référentiels pour le futur (visions, projections, anticipations, etc.). 
Ces éléments sont importants à préciser, car il est vraisemblable qu'ils expliquent en grande partie la confusion (indifférenciation) qui règne le plus souvent entre la GRH et le management global de l'entreprise en l'absence d'un modèle général de référence explicite pour la GRH.

\section{La confusion fréquente entre les trois niveaux de la GRH et celui du management (l'indifférenciation)}

L'analyse détaillée des activités rentrant dans le champ de la GRH nous a conduit à distinguer :

- le niveau « 1 » de l'administration du personnel, lorsqu'il s'agit d'appliquer, d'une part, la législation du travail, la réglementation, les conventions collectives et, d'autre part, les directives de la direction sous la forme de procédures préalablement définies ;

- le niveau « 2 » des politiques du personnel, lorsqu'il s'agit de décider des principales orientations concernant le personnel et touchant à l'emploi, aux rémunérations, à la valorisation ou à la participation. Ce niveau de problème est le plus souvent couvert par la direction (partiellement par un responsable délégué);

- le niveau « 3 » de la stratégie de GRH, lorsqu'il s'agit de regrouper l'ensemble des choix d'organisation et de management concernant les hommes en rapport avec les décisions portant sur les orientations stratégiques relatives aux modes de production, aux technologies, aux produits et aux marchés de l'entreprise.

Ces trois niveaux, ici nettement différenciés, sont souvent imbriqués, voire amalgamés, dans l'esprit du dirigeant et cela d'autant plus que les trois dimensions (administrative, politique et stratégique) sont souvent présentes dans de nombreuses problématiques (elles se conjuguent et s'entremêlent).

Cette difficulté de différenciation entre les trois niveaux conduit de nombreux dirigeants à considérer la GRH comme relevant du premier niveau (administratif) à caractère réducteur, et à s'approprier ainsi les deuxième et troisième niveaux dans une fonction commune de direction générale qualifiée de management ${ }^{8}$. Cela contribue de fait à souligner l'importance que le dirigeant attribue aux hommes même si c'est au détriment d'une définition englobante et savante de la GRH.

Paradoxalement, cette confusion n'est pas pour autant synonyme de contreperformance sociale si on en juge par le climat qui règne dans de nombreuses PME

8. Plusieurs enquêtes de terrain réalisées en France par nos soins de 1987 à 1997 ont confirmé cet état de fait. 
(à perception peu différenciée). En effet, le processus de différenciation croissant qui est en relation avec la taille, signe d'une plus grande compréhension, n'est pas toujours le gage d'une plus grande maîtrise de la GRH comme cela peut être constaté dans des unités de grandes dimensions.

\section{Le manque de référence à un modèle global de régulation de la GRH chez le dirigeant}

En corollaire du constat de confusion entre niveaux, signalé précédemment, on note l'absence d'une modélisation ou, plus simplement, l'absence d'une représentation complète et complémentaire des principaux axes d'actions de la GRH ainsi que de l'anticipation de leurs principaux effets dans une perspective de commande et de régulation (absence fréquente d'une vision systémique globale). Ce constat réalisé depuis plusieurs années nous a conduit à proposer l'architecture d'un système de GRH intégré (Mahé de Boislandelle, 1998) s'appuyant sur un «mix social» ou panoplie de domaines d'actions complémentaires au plan de la gestion des personnels. Ce mix social concerne principalement l'emploi, la rémunération, la valorisation et la participation couvrant ainsi tous les domaines de la GRH. Ces actions produisent différents effets directs et indirects repérables par des pôles de résultats.

Les effets directs portent respectivement sur l'effectif, la masse salariale, les niveaux et les types de compétences et le contexte relationnel.

Les effets indirects sont appréhendés sur le plan du climat social, de l'image sociale et des performances technico-économiques.

\section{Comportements au travail du dirigeant}

Les comportements au travail (activités) des dirigeants de PME ou caractéristiques relatives à leurs pratiques du management au vu de plusieurs enquêtes sur leur emploi du temps (Al Ali Adday, 1997) sont les suivants :

- un rythme soutenu de travail ;

- des activités brèves, variées et fragmentées;

- un goût prononcé pour l'action ;

- le recours fréquent à la communication verbale (téléphone, face à face, réunions, etc.);

- la confection et l'utilisation d'un réseau de contacts internes et extérieurs à l'organisation (network);

- la gestion d'un mélange de droits et de devoirs ;

- un rôle fréquent d'initiateur;

- une situation de solitude (dans la décision) variable selon les étapes de développement de l'entreprise et la nature des décisions à prendre (le plus souvent relativement faible). 
Ces activités et comportements, dont la connaissance est essentielle, sont souvent affectés par des biais de perception qui sont liés à la taille, à la multitude des problèmes à résoudre et notamment aux phénomènes d'urgence.

\section{Cognition et biais de perception chez le dirigeant}

En nous référant au système cognitif du dirigeant, nous entendons moins la réalité «objective» dans laquelle il se trouve que la construction subjective qu'il réalise de sa propre réalité, en supposant bien sûr que c'est à partir d'elle qu'il décide et agit.

On peut admettre, en effet, que c'est une structure cognitive qui guide le dirigeant, comme tous les individus, dans les actions qu'il entreprend et dans l'interprétation des événements.

Les activités et comportements du dirigeant de PME (voir ci-dessus) combinés à la multitude des problèmes rencontrés dans le quotidien se traduisent par des confusions de perception sur au moins trois plans : l'horizon de calcul (CT, MT, LT), l'importance relative des opérations (stratégique / opérationnel) et le maniement des événements (tactique, stratégique).

\section{L'horizon de calcul}

Définis de manière rationnelle par des chercheurs et théoriciens de la gestion, les horizons de calcul des dirigeants en matière de GRH sont souvent entremêlés. Bien que soit le plus souvent privilégié le court terme, on constate à la réflexion que celui-ci est souvent porté à durer. Il se transforme alors en moyen ou en long terme par adaptations successives et négociations internes avec le personnel intéressé (c'est le cas, par exemple, dans la gestion des carrières au cas par cas, des programmes de formation renouvelés, etc.). Cela est bien sûr révélateur de certaines carences dans le domaine de l'anticipation.

\section{L'importance des opérations et des actions}

Comme pour l'horizon de calcul, les décisions touchant au personnel peuvent avoir une importance variable. Par exemple, la décision de formation d'un salarié dans un domaine précis (acquisition d'une technique de travail nouvelle, etc.) peut être interprétée comme ayant un sens limité de régulation locale. En réalité, cette formation peut conduire le dirigeant à réorienter son mode de production ou à susciter de nouvelles activités, etc. En bref, ce qui pourrait être du domaine du local, du «correctif» dans une grande organisation peut avoir une importance stratégique en PME. Cela nous conduit à déclarer que « tout est stratégique en PME ».

Le recrutement ou le départ d'un salarié peut être stratégique. Par exemple, sur un effectif limité, le départ d'un salarié ou l'entrée d'un nouveau va modifier fortement la proportion en personnel. Dans une micro-entreprise de cinq salariés, un salarié absent 
représente à lui seul $20 \%$ d'absentéisme, etc. Un conflit avec le seul représentant des salariés va entraîner une perturbation dans l'ensemble de l'organisation.

Nous appelons ce phénomène lié aux proportions l'effet de grossissement (Mahé de Boislandelle, 1996b ; 1996c). Il caractérise les effets amplifiants des changements portant sur des petits nombres. Ce même effet peut se conjuguer avec d'autres effets résultant de perceptions déformantes (dramatisation, ignorance, hypertrophie, etc.) liées au manque de recul que l'on peut qualifier d'effet de microcosme.

Dans le même ordre d'idées, on observe des interférences entre des actions à caractère tactique et à caractère stratégique lorsqu'il s' agit de ressources humaines. Par exemple, la mutation d'un salarié d'un service à un autre, insignifiante dans une grande entreprise, peut modifier la structure de l'organisation et donc la dynamique d'une PME dans la mesure où celle-ci est fortement sensible à certains changements de faible amplitude. Dans cet esprit, la non-prise en compte, par

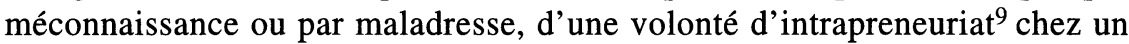
cadre peut conduire celui-ci à quitter l'organisation par manque d'avenir ou de possibilité d'épanouissement. Ce genre de départ est souvent dommageable pour l'entreprise, car il peut susciter l'émergence de nouveaux concurrents issus de l'entreprise elle-même.

Ces constatations liées au mode de travail et aux types de perceptions des dirigeants sont marquantes de spécificités du management en PME et éclairantes du système de GRH adopté. Elles sont des corollaires du système cognitif du dirigeant et de son comportement au travail.

$\mathrm{Au}$ vu des nouveaux éléments relatifs aux activités et aux biais de perception que nous avons intégrés, il est possible de donner une représentation enrichie et complémentaire des déterminants du système de GRH en PME.

Au-delà des caractéristiques de la direction, fortement déterminantes du système, il reste à préciser d'autres paramètres distinctifs de la GRH en PME qui sont liés à la taille et aux relations de dépendance de l'organisation.

\section{2. Émergence organisationnelle de la fonction RH en relation avec les changements de taille et les réseaux de dépendance de l'entreprise}

Parmi les autres déterminants du système de GRH en PME, une place particulière doit être accordée aux changements de taille (allant de 10 à 500 salariés) et aux situations de dépendance amont (à l'égard des fournisseurs) ou aval (à l'égard des clients) auxquels l'entreprise doit faire face.

9. Volonté de prendre des initiatives de type entrepreneurial dans le cadre d'une mission se situant dans l'entreprise. 
FIGURE 4

Déterminants directoriaux du système de GRH

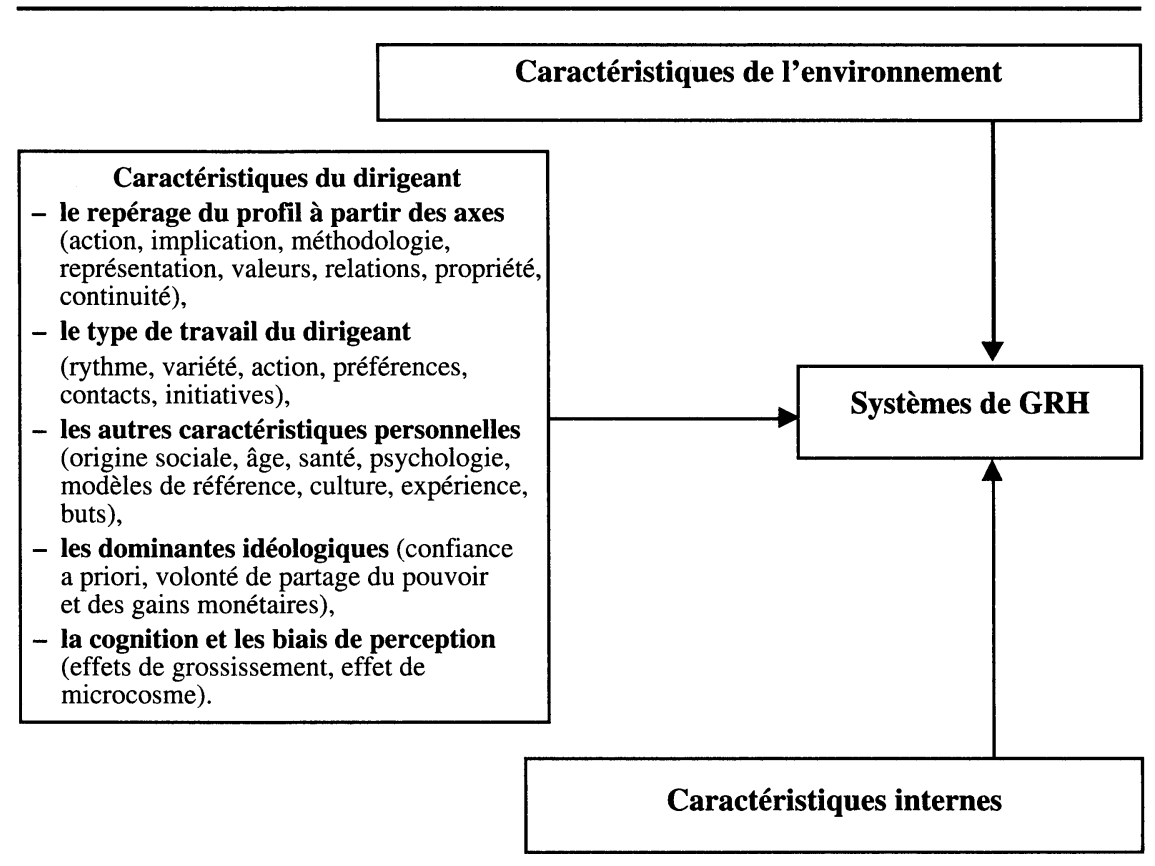

\section{Formalisation et professionnalisation croissante de la GRH avec la taille}

Différentes études empiriques conduites par nous-même ou par d'autres auteurs font apparaître une évolution de la prise en charge en fonction de la taille qui s'inscrit globalement dans ce qu'on peut appeler un processus général de formalisation.

Ce processus peut se résumer par les caractéristiques suivantes :

- émergence progressive d'un service du personnel ou de ressources humaines dans la tranche de 50 à 100 salariés, plus largement et de façon systématique au-delà de 150 ;

- processus croissant de délégation de responsabilités dans certains domaines de la GRH essentiellement dans le champ de l'administration du personnel (respect des directives juridiques, calcul de la paie, etc.) et plus parcimonieusement dans le champ des politiques de personnel (formation, appréciation du personnel, gestion des effectifs, etc.).

L'émergence d'un DRH (directeur des ressources humaines) à plein temps est réservée aux plus grandes PME (sauf exception). On observe en revanche 
l'apparition progressive de responsables du personnel occupés à des tâches relevant de la gestion du personnel pour $25 \%, 50 \%$ et $75 \%$ de leur temps.

Parallèlement au processus de formalisation, la professionnalisation de la GRH augmente avec la taille.

La maitrise de différentes tâches d'administration du personnel exige des connaissances en informatique (emploi de logiciels ouverts à des tâches de plus en plus larges dans le champ des ressources humaines), en droit, en psychologie, en communication, etc.

La maîtrise des politiques de personnel exige, de son côté, 1) l'adoption de procédures de gestion des effectifs, des rémunérations, de la formation et de la participation (communication...) et 2) l'importation d'outils (utilisés par les grandes entreprises) de diagnostic, de gestion prévisionnelle, d'appréciation du personnel, de classement de postes, etc.

Dans les plus grandes PME, on a fréquemment recours à des tableaux de bord sociaux et à des formes de contrôle de gestion sociale.

Avec l'accroissement de la taille, on observe enfin un processus d'internalisation de différentes activités auparavant sous-traitées. Plus exactement, certaines activités sont internalisées alors que d'autres sont externalisées.

\section{Pratiques importées et pratiques induites par certaines dépendances}

Si le rôle endogène joué par le dirigeant, au regard de la conception de la GRH, de l'adoption d'un système et de la mise en pratique d'outils et de procédures ne fait aucun doute, il est nécessaire, pour rendre compte fidèlement de l'état des pratiques effectives, de considérer les facteurs exogènes influant sur les choix de GRH.

Parmi les facteurs exogènes, nous mentionnerons les pratiques importées :

- par le recours à des consultants en GRH (conseillers) ou par le recours à la sous-traitance de certaines activités de GRH (traitements informatiques de la paie, contentieux avec le personnel, etc.);

- par le biais de formations patronales assurées par des pairs et dispensées aux dirigeants sous forme de recherches de solutions en groupe (problem solving);

- par l'acquisition d'outils standardisés de GRH et de plus en plus à la portée des PME tels que des logiciels de paie, de gestion des effectifs, de gestion de la formation, de gestion de fichiers, de tableurs, etc., qui facilitent l'appréhension de plus en plus analytique des données humaines et sociales;

et les pratiques induites :

- par certaines formes de coopérations contractuelles entre PME (entreprises en réseau, structuration par franchise, etc.); 
- par des relations de sous-traitant à donneur d'ordres ; le donneur d'ordres assurant un rôle moteur d'acheteur ou de prescripteur suivant un cahier des charges exigeant en termes de qualité et de prix (délais, stocks, tolérances, défauts, etc.).

Concernant les pratiques induites, différents travaux de recherche ont souligné le rôle fortement inducteur, dans le champ de la GRH, d'une politique de certification (ISO 9000, 9002, etc.) ou d'assurance qualité... imposée par le donneur d'ordres au faisceau de ses sous-traitants fournisseurs (Laval, 1994).

C'est ainsi que parmi les mix sociaux induits par une politique de qualité imposée par l'impartiteur (donneur d'ordres), il ressort que les politiques de valorisation et de participation sont les plus directement affectées.

Par exemple, la formation à la qualité totale (TQC) engendre :

- d'une part, l'analyse, la prévention, le contrôle des opérations, la sensibilisation aux résultats (dysfonctionnements), etc. ;

- et, d'autre part, l'induction d'un système de coopération vigilante entre services. Chaque service doit se percevoir comme un sous-système à la fois client et fournisseur des services amont et aval.

Dans le cadre de la sous-traitance communautaire ${ }^{10}$, les actions de soumissionnement, réalisées par des entreprises associées momentanément pour répondre à un appel d'offres, induisent l'adoption de politiques de production qui créent de forts besoins de coordination et notamment d'harmonisation des politiques de personnel.

\section{Conclusion}

Contrairement à certains a priori, la GRH en PME n'est pas simple ; la complexité n'est pas l'apanage des seules grandes organisations. Les différents modèles de déterminants examinés précédemment en donnent la mesure.

L'incidence particulièrement forte de certains déterminants, externes ou internes, doit permettre après analyse d'anticiper certaines conséquences afin d'améliorer les performances sociales du système.

À l'évidence, certains outils de GRH utilisés en grande entreprise sont adaptables ou transposables en PME. Il peut en être ainsi de certains indicateurs ou procédures, et notamment de la plupart des procédures analytiques à dimension qualitative (à l'exception de ce qui privilégie les grands nombres).

10. Assurée par des entreprises associées momentanément. 
En revanche, la PME n'étant pas un modèle réduit de la grande entreprise, certains outils sont à concevoir et à mettre à la disposition du dirigeant en raison de particularité dont la prise en considération est essentielle. L'effet de grossissement et de microcosme qui résultent de son système cognitif doivent donc être corrigés, autant que faire se peut, pour réduire les risques de distorsion.

Plus encore, l'efficacité n'est pas toujours du côté de la grande entreprise. C'est ainsi que certaines pratiques et procédures utilisées ou expérimentées en PME sont susceptibles d'être transférées avec bonheur en grande entreprise. La démarche de PMIsation adoptée par de nombreuses grandes entreprises lors d'opérations de réorganisation est là pour le confirmer. Cette dernière est souvent considérée et vécue comme une transformation organisationnelle génératrice d'une plus grande efficacité.

Les raisons en sont que les indifférenciations formelles de la PME ne sont pas forcément synonymes de contre-performance au regard de la gestion en général et de la GRH en particulier. Plus précisément, certaines faiblesses opératoires (modestie des outils) semblent largement compensées par l'obtention d'un contexte relationnel qui favorise les coopérations entre acteurs et qui se révèle donc au total beaucoup plus positif.

En d'autres termes, ce qui est organisé et formalisé n'est pas inéluctablement plus efficace que ce qui n'est pas formalisé et différencié de manière immédiatement repérable.

La formalisation qui est un moyen de lutte contre le désordre d'un système (l'entropie) cesse d'être nécessaire lorsqu' on maintient la taille d'un système à une échelle maîtrisable.

Certaines qualités associées au petit nombre telles que l'absence d'anonymat, la convivialité, la multiplicité des échanges, etc., permettent de compenser certaines insuffisances de gestion de la PME comparativement à la grande entreprise.

Le «small» n'est pas toujours «beautiful», mais la GRH en PME n'est pas toujours pire que celle qui est pratiquée en grande entreprise si on considère l'ensemble des axes du mix social et surtout les effets.

Plus encore, la richesse de l'informel associée à la diversité, à la flexibilité et à la solidarité peut parfois compenser la « rigidité » de certains systèmes formels peu susceptibles de se remettre en cause.

Si les PME ont beaucoup à apprendre des GE, il n'est pas moins sûr que réciproquement les GE n'aient à tirer des PME beaucoup d'enseignements pour atteindre une plus grande efficacité économique, organisationnelle et sociale.

L'examen des pratiques en PME associé à la recherche et à la réflexion dans ce domaine peuvent engendrer des modèles exportables vers les grandes organisations. Certaines logiques de réorganisation et d'animation actuelles conduisent à en être de plus en plus convaincu. 


\section{Bibliographie}

AL ALI ADDAY, S. (1997), Les managers en activité : recherche empirique, Thèse en sciences de gestion, IAE-ESUG, Université Toulouse I, février.

COSSETTE, P. (1993), « La vision stratégique du propriétaire-dirigeant de PME », Actes du colloque de Tunis, octobre.

GREGO-GRH (1994), « Annuaire GRH en PME », Document de travail, Montpellier, avril.

FILlion, C.J. (1991), Visions et relations : clef du succès de l'entrepreneur, Montréal, Les Éditions de l'Entrepreneur.

GARAND, D. (1993), «Les pratiques de gestion des Ressources Humaines en PME : une synthèse conceptuelle et empirique », Trois-Rivières, GREPME, mars.

LAVAL, F. (1994), La gestion des ressources humaines des entreprises fournisseurs partenaires : l'impact de la politique d'achat d'un grand groupe industriel, Thèse de sciences de gestion, IAE de Montpellier, janvier.

GASSE, Y. et C. CARRIER (1992), Gérer la croissance de la PME, Montréal, Les Éditions de l'Entrepreneur.

MaHÉ De Boislandelle, H. (1993), Gérer les hommes de la jeune entreprise, Paris, Éditions Chotard.

MAHÉ DE BoISLANDELLE, H. (1995), « Spécificités des problématiques de recherche GRH en PME », Revue Gestion 2000, n 1, janvier-février.

MAHÉ DE BOISLANDELLE, H. (1996a), «L'après-licenciement économique et l'après-plan social. Conséquences sur les politiques de GRH et sur l'implication du personnel restant », juin (en collaboration avec C. Fabre et A. Briole), Rapport FNEGE /AGRH, 98 pages.

MahÉ De Boislandelle, H. (1996b), «L'effet de grossissement chez le dirigeant de PME », Revue Gestion 2000, vol. 4, juillet-août.

MAHÉ De BoISLANDELle, H. (1996c), «L'effet de microcosme en PME », CIFPME, TroisRivières, UQTR, octobre.

MAHÉ DE BOISLANDELLE, H. (1996d), «L'implication des salariés restants après un plan social », 6e Congrès de l'AGRH, Université de Paris-Assas, novembre.

MAHÉ DE BOISLANDELLE, H. (1997), Management des organisations de services sportifs, chapitre III : « De l'organisation efficace au management des ressources humaines », Paris, Presses universitaires de France, avril.

MahÉ De Boislandelle, H. (1998), Dictionnaire de gestion, Paris, Economica, janvier, 500 pages.

Mahé De Boislandelle, H. (1998), Gestion des Ressources Humaines dans les PME, $2^{\mathrm{e}}$ édition revue et augmentée, Paris, Economica, janvier, 490 pages.

MinTZBERG, H. (1976), «Que fait un dirigeant dans sa journée ?», Revue Harvard/L'expansion, $\mathrm{n}^{\circ} \mathrm{l}$, Paris. 